\title{
GÉNERO Y CONFLICTO EN LAS RELACIONES DE PAREJA HETEROSEXUALES: LA DESIGUALDAD EMOCIONAL
}

\section{Ana Dolores Verdú Delgado}

anad.verdu@yahoo.es Universidad Miguel Hernández de Elche

Recibido: 06-02-2013

Aceptado: 22-03-2013

\section{Resumen}

Este artículo explora, a partir de los resultados obtenidos en un trabajo de tesis doctoral, el papel del género en la manifestación del conflicto en parejas heterosexuales, poniendo el acento en la desigualdad emocional como origen de insatisfacción amorosa de las mujeres y en la forma en que la resolución del conflicto refuerza a su vez los roles de género tradicionales. La información en este análisis proviene concretamente de 46 entrevistas en profundidad, realizadas a 23 mujeres y 23 hombres, con quienes se contactó a través de distintos centros universitarios de la provincia de Alicante.

Palabras clave: pareja heterosexual, género, conflicto, desigualdad emocional.

\begin{abstract}
This article explores, from the results obtained in a PHD thesis, the role of gender in the expression of conflict in heterosexual couples, with a focus on emotional inequality as the origin of women's dissatisfaction with love and on the way in which conflict resolution eventually reinforces traditional gender roles. The information in this analysis arises specifically from 46 in-depth interviews with 23 women and 23 men, who were contacted through different university centres in the province of Alicante (Spain).
\end{abstract}

Key words: heterosexual couple, gender, conflict, emocional inequality. 


\section{Introducción}

En los últimos años se ha despertado un interés por parte de las Ciencias Sociales y de los Estudios de Género por el modo en que la desigualdad de género se expresa en las relaciones de pareja heterosexuales a partir de la observación de que, si bien la igualdad jurídica ha logrado reducir considerablemente la vulnerabilidad social de las mujeres, las relaciones entre hombres y mujeres siguen estando notablemente influidas por los valores que tradicionalmente han mantenido una cultura patriarcal y sexista, generando una situación de desequilibrio en el modo en el que se experimentan y organizan las relaciones afectivas entre los sexos.

Autoras como Anna G. Jónasdóttir consideran las relaciones amorosas heterosexuales el campo de estudio central para la teoría feminista del siglo XXI por la forma en que reproducen la desigualdad mediante la normalización de un intercambio asimétrico entre hombre y mujer. Este planteamiento no contemplaría la igualdad únicamente como situación derivada de la distribución equitativa de las tareas domésticas en la convivencia o de la presencia de autonomía económica por parte de la mujer, sino que otorgaría una importancia clave a los aspectos que tienen que ver con las inversiones de energías vitales que se producen en las relaciones interpersonales, y a los beneficios obtenidos con ellas. Por otro lado, el análisis de la interacción emocional entre hombre y mujer en sus relaciones afectivas desde un enfoque estructural de género lleva a contemplar la pareja heterosexual como un espacio sujeto a la contradicción, por la convergencia que en él tienen la libertad e igualdad que definen el modo de vida actual y los valores y prácticas que generarían una experiencia cotidiana atravesada por la desigualdad.

Partiendo de esta apreciación, este artículo presenta algunos de los resultados obtenidos en el trabajo de tesis doctoral "El amor y las relaciones de pareja desde la perspectiva de género. Un estudio interdisciplinar" (Verdú Delgado, 2012), particularmente aquéllos que surgen del análisis de la relación entre género y conflicto en parejas heterosexuales. Los resultados aquí tratados apuntarían a una interferencia negativa del sistema tradicional de género en la dinámica de las parejas heterosexuales en la actualidad, expresada con especial fuerza en el manifestación del conflicto en torno a la desigualdad emocional, así como en una tendencia al reforzamiento de los roles de género como respuesta al conflicto.

\footnotetext{
${ }^{1}$ Tesis dirigida por Anastasia Téllez Infantes y Carmen Mañas Viejo, defendida el 11 de enero de 2013 en la Universidad Miguel Hernández de Elche.
} 


\section{Desigualdad de género en la pareja heterosexual. Aportaciones desde las Ciencias Sociales y el Feminismo}

A pesar de las transformaciones sociales que han dado lugar en las sociedades democráticas a una nueva concepción de las relaciones de amor y de pareja, a las que se han hecho extensibles valores como la igualdad, la libertad y los derechos individuales, muchos estudios advierten de las múltiples constricciones que los modelos culturales imponen a los modos en que hombres y mujeres se relacionan, limitando sus capacidades para el desarrollo efectivo de una interacción entre los sexos realmente libre e igualitaria. De ahí que el análisis de la desigualdad en este contexto sea especialmente complejo y exija prestar atención al modo en que se mantiene desde la propia actuación del individuo, señalando la importancia que tiene la interiorización de la identidad de género. La adquisición de una identidad sexuada que ya incorpora en sus valores y características una interpretación de las relaciones entre los sexos más o menos jerárquica se considera desde este punto de vista uno de los mecanismos más eficaces de reproducción de un determinado sistema de género. Este aspecto hace que la desigualdad entre hombres y mujeres en la actualidad se analice haciendo hincapié en su dimensión simbólica (Bourdieu, 2000) y que refleje un intento de trascender los modelos teóricos que pueden llegar a aniquilar la voluntad de las mujeres mediante una articulación del patriarcado que gire únicamente alrededor del dominio masculino (Jónasdóttir, 1993: 273; Izquierdo, 1998: 118).

Al hacer referencia al conjunto de creencias y prácticas que producen una relación entre los sexos atravesada por el poder, autoras/es como Janet Saltzman (1992) señalarían la división sexual del trabajo dentro y fuera de la familia como primer blanco del cambio o de la estabilidad del sistema de desigualdad de género. En particular, la división sexual del trabajo dentro del hogar, también entendida como dinámica de intercambio conyugal, hace referencia a procesos que ocurren en el nivel micro de la realidad en los que el componente de voluntariedad está mucho más presente que en los procesos macroestructurales. Lo que Saltzman propone es que es la vinculación de procesos de los distintos niveles (micro, medio y macro) lo que mantiene el sistema de desigualdad estable. Su teoría apoya la visión de Curtis (1986) desde la cual los hombres adquieren un mayor poder interpersonal en sus relaciones conyugales con las mujeres, ayudados por un contexto en el que la autoridad constituye un privilegio simbólico masculino y por el hecho de que, en general, los hombres todavía disponen de mayor poder económico que las mujeres. Ambas ventajas en la dinámica de la relación heterosexual reforzarían en las mujeres el rol de cuidadoras a través de un intercambio conyugal difuso entre seguridad material y cuidados.

Desde estas premisas, la relación de pareja heterosexual puede estudiarse como interacción de naturaleza micro en la que se manifiestan y actúan inconscientemente expresiones del sistema social y cultural en el que acontecen, reproduciendo el orden jerárquico que impera a nivel macroestructural. 
Otra forma de abordar la interacción de pareja es valorando en mayor medida la influencia que las experiencias interpersonales pueden ejercer sobre el sistema, poniendo énfasis así en el carácter dinámico de los fenómenos sociales. Esta visión sería defendida por Anthony Giddens (1995), quien analiza la transformación de los modelos de relación de pareja a partir de la democratización de las relaciones personales como efecto de la prevalencia de la intimidad y la satisfacción individual sobre otros aspectos ligados a las convenciones sociales. Este cambio sustancial en las formas de amor adoptadas en la actualidad apoyaría una mayor igualdad entre los sexos, y a la vez, llevaría implícita una mayor fragilidad de las relaciones de pareja, sometidas a una necesidad constante de validación (Giddens, 1995; Bauman, 2005). En este sentido, también resultan relevantes las aportaciones de Ulrich Beck y Elizabeth BeckGernsheim (2006), quienes valoran la colisión de intereses que produce la experiencia simultánea de necesidad de amor y de libertad, observando el modo en que las nuevas libertades, obligaciones y conflictos intervienen y moldean las relaciones amorosas. Así, el triunfo de la individualización habría liberado a los seres humanos de los roles de género, haciendo posible que la pareja heterosexual se idealice como proyecto personalizado de amor, y destapando a su vez la existencia de un conflicto específico en torno al deseo de equidad de las mujeres en un escenario que todavía asocia lo femenino a las mayores responsabilidades con respecto a las relaciones interpersonales y el hogar. Estas observaciones ponen de relieve que, si bien la democracia acarrea una valoración positiva de las relaciones de pareja igualitarias, en la práctica, la vida personal reflejaría, junto con la pérdida de fuerza de los roles de género tradicionales, la falta de asimilación de una cultura democrática por parte de los individuos (Giddens, 1995).

De hecho, según otras voces críticas, la forma en que está cambiando la idea del amor y las expectativas individuales con respecto a las relaciones de pareja podría no estar transformando sustancialmente el modo en que mujeres y hombres actúan en una relación con mayores responsabilidades y negocian sus papeles en la vida cotidiana (Jamieson, 1999). Contestando a Giddens, Jamieson sostiene que la experiencia real de los individuos, en sus papeles de amantes, parejas, madres, padres y amigos, estaría más determinada por los estereotipos de género que sostienen las viejas desigualdades que por el nuevo ideal de igualdad. Este matiz haría visible la posible influencia del sistema de género en la expresión del conflicto en las relaciones íntimas heterosexuales, por encima de la indeterminación, la fragilidad y la tensión consecuentes de la irrupción de la libertad y la búsqueda de satisfacción personal en el terreno de la pareja.

Además, para las Ciencias Sociales el estudio de las emociones ha dado lugar a corrientes teóricas que, desde el reconocimiento de esta dimensión humana, han avanzado en la comprensión de los fenómenos sociales y, al mismo tiempo, han convertido el universo emocional en un objeto de análisis en sí mismo, desvelando sus interacciones con la estructura social (Bericat, 2000). Dentro de estas corrientes la obra de Arlie R. Hochschild (1979, 1997) se considera fundamental para entender cómo la cultura interviene en la experiencia y expresión de las emociones. Hochschild observa que una de las ideologías con mayor impacto 
en el modo en que los individuos se comportarán con respecto a éstas, obedeciendo las normas emocionales, es la que proviene del sistema de género, notando que la prescripción de comportamientos femeninos/masculinos estaría detrás de la tendencia a que sean las mujeres quienes asuman una mayor responsabilidad sobre los trabajos de cuidado y sobre aquéllos que implican generalmente una mayor gestión de las emociones.

La teoría de Hochschild aparece reflejada en los resultados de las investigaciones que en los últimos años han detectado la presencia de un sistema de intercambio emocional asimétrico en el contexto de la relación de pareja heterosexual (Hite, 1995; Duncombe y Marsden, 1993; Fishman, 1982; Benjamin, 2011). Al mismo tiempo, estos mismos estudios coinciden en señalar la falta de satisfacción de la población femenina con respecto a este intercambio asimétrico de cuidados, aspecto que contemplan como uno de los rasgos que caracterizarían las relaciones afectivas entre hombres y mujeres en la actualidad.

La idea de que las identidades de género encierran a las mujeres en el rol de dadoras/cuidadoras y de que este hecho forma parte de la desigualdad estructural entre hombres y mujeres que sobrevive con más fuerza en la sociedad contemporánea es también la tesis de Shere Hite (1988), cuya obra ofrece un análisis detallado de las entrevistas que realiza a 4.500 mujeres veinte años después de la llamada "revolución sexual", con la intención de comprobar el cambio efectivo hacia un modelo igualitario y libre de relaciones de pareja. Las conclusiones a las que esta investigación llega le permiten hablar de la existencia de una "violencia emocional" que, mediante la negación del derecho de las mujeres a ser amadas en las mismas condiciones en las que son amados los hombres, estaría provocando en ellas la destrucción de la autoconfianza y elevados niveles de insatisfacción.

En su trabajo Mujeres y amor. Nuevo informe Hite, la autora utiliza el término "contrato emocional" para describir lo que en su opinión constituye una ideología a espaldas del sistema, es decir, el funcionamiento de una estructura social asimétrica en lo referente a los afectos que permite al hombre constituirse en mayor medida como beneficiario e inculca en la mujer el rol de dadora, todo ello en perfecta compatibilidad con un sistema político y social basado en la igualdad de derechos de todos los individuos (Hite,1988: 80). Hite señala a su vez esta desigualdad emocional como base fundamental del conflicto en las relaciones heterosexuales.

"Pero esa interacción emocional es perturbada por una serie de actitudes subliminales, confusas, degradantes orientadas hacia el sexo del cada uno; presunciones entretejidas en nuestras ideas de quién es el hombre y quién la mujer. Creemos que la mujer es "amante y dadora" mientras que el hombre es "hacedor", y que el uno tiene más derechos que la otra. [...] La desigualdad emocional es la base fundamental que bloquea el amor en unas relaciones" (Hite, 1988: 118-120).

Como otras investigadoras, Hite relaciona la asimetría en la interacción emocional entre mujeres y hombres con la extensión de dos culturas del amor. La ideología del amor como esencia, propia de la feminidad, haría que las mujeres asumieran la relación amorosa con el hombre con el cuidado y la atención que requiere una labor profesional, intentando ajustar la realidad al nivel de sus expectativas, mientras que la basada en la represión del amor, o ideología masculina, según Hite, dificultaría la entrega afectiva del hombre, al asociar el amor 
con la debilidad e incentivar su represión con el objetivo de alcanzar un ideal de masculinidad orientado a la autosuficiencia. Esta divergencia en las formas en que la sociedad transmite modelos culturales en torno a las emociones estaría estrechamente vinculada con el sexismo y es vista generalmente como generadora de problemas vinculados con las expectativas y niveles de satisfacción de los individuos en sus vidas afectivas (Hite, 1995; Sanz, 2000; Rebollo, 2006).

Otro de los trabajos que se centran en la desigualdad emocional como origen de un conflicto estrechamente relacionado con el sistema de género es El poder del amor de Anna G. Jónasdóttir. Esta autora opina que la raíz de la desigualdad entre hombres y mujeres en el mundo contemporáneo yacería en el intercambio asimétrico de fuerzas vitales, y con ello pone el acento en el amor, no solamente como aspecto ligado a las emociones, sino entendido como conjunto de "prácticas socio-sexuales" ligadas a un proceso creativo que nos constituye como cuerpos y mentes (Jónasdóttir, 1993: 21-49), señalando que, si bien "existe la igualdad en cuanto a derechos legales y oportunidades desde un punto de vista formal, también se dan ciertos mecanismos subyacentes que recortan las posibilidades reales de las mujeres para alcanzarlos" (Jónasdóttir, 1993: 13).

La postura de Jónasdóttir (1993: 48) lleva a señalar la interacción íntima e informal entre los sexos como espacio privilegiado para el análisis social y de género. En su opinión, la desigualdad de género se reproduce hoy a través de la "producción de vida", o del vínculo que nos une como seres sexuales y dependientes. Este tipo de producción que nos crea, nos mantiene y asegura nuestro bienestar cotidiano sería comparable a cualquier otra producción (haciendo una analogía con el modo productivo capitalista), ya que también se regula mediante mecanismos como la acumulación, la explotación o la distribución no recíproca con los que los seres humanos construirían una determinada estructura de poder. Es decir, el desequilibrio en la interacción emocional entre mujeres y hombres debe ser analizado como aspecto consecuente de la desigualdad estructural de género, constituyendo un fenómeno relacionado, como indica Jónasdóttir, con la mayor autoridad masculina, frente a la influencia femenina, que extendida a la interacción íntima, permitiría el establecimiento normalizado de relaciones de pareja no recíprocas en términos de energía dedicada. Junto a este aspecto, Jónasdóttir también resalta la forma en que la disponibilidad de las mujeres para la satisfacción de las necesidades de los hombres está presente en los mecanismos de socialización, así como el derecho de los hombres a reservar sus energías para ellos mismos. Desde esta perspectiva, la disponibilidad de amor y cuidado femenino que la sociedad reserva a la población masculina sin exigirle la misma dedicación en el intercambio de energía con las mujeres se traduciría en una "acumulación de fuerzas existenciales" que el hombre podría utilizar para mantener su autoridad histórica. "Los hombres pueden configurarse como seres sociales poderosos y continuar dominando a las mujeres a través de la acumulación constante de las fuerzas existenciales tomadas y recibidas de las mujeres. Si el capital es la acumulación de trabajo alienado, la autoridad masculina es la acumulación de amor alienado" (Jónasdóttir, 1993: 53). 
En resumen, el grado de igualdad entre hombres y mujeres debe analizarse, no sólo a partir de los elementos estructurales o institucionales característicos de nuestro contexto cultural en la actualidad, sino también, y muy especialmente, a partir de los valores que interfieren en la producción de prácticas femeninas y masculinas, así como en los intercambios que tienen lugar entre mujeres y hombres en el contexto de sus relaciones íntimas.

\section{Metodología}

\subsection{Objetivos e hipótesis}

La tesis de la que parte este artículo tiene como objetivo principal el análisis con enfoque interdisciplinar de las condiciones que envuelven las relaciones de pareja heterosexuales en relación con la categoría género, poniendo el acento en algunos aspectos particulares como son los conflictos y la satisfacción, en una población escogida a partir de su vinculación con la universidad en la provincia de Alicante $^{2}$. Valorando este objetivo básico, el trabajo plantea un acercamiento al papel que tienen los valores y estereotipos de género que la cultura transmite con relación al amor y la pareja, y a la forma en que estos valores producen, reproducen o resuelven los principales conflictos en la dinámica de la relación heterosexual, en un contexto de democracia, libertad e igualdad formal.

En este texto se recogen específicamente algunos de los resultados obtenidos en el análisis del conflicto, en especial, aquéllos que hacen alusión a la desigualdad en la interacción emocional y al modo de resolver los conflictos. Los resultados y las conclusiones aquí expuestas se articulan dentro de unas líneas de investigación definidas que dieron forma a una serie de hipótesis de trabajo estructuradas alrededor de algunos conceptos que en la actualidad centran una atención especial por parte de los Estudios de Género:

1- Las relaciones de pareja están atravesadas por un sistema cultural de influencia patriarcal y reproducen en consecuencia una estructura de desigualdad. En este sentido, se aprecia que los intercambios en una relación íntima pueden estar mucho más influidos por los aspectos culturales y subjetivos incorporados en la propia identidad masculina o femenina que por los principios y valores que regulan nuestra convivencia como sociedad.

\footnotetext{
${ }^{2}$ Como parte de los objetivos de esta tesis también se incorpora el interés por la violencia contra las mujeres en la relación de pareja, por tratarse de un fenómeno social ligado a los valores y dinámicas generadas por un determinado esquema de género, y por constituir un problema que reclama especial atención por parte de la sociedad. Los resultados relativos a la violencia no se han contemplado en este artículo.
} 
2- En coherencia con la pervivencia de un esquema tradicional de género, el aprendizaje de la feminidad puede seguir premiando ciertas formas de sacrificio y altruismo en lo referente al amor por parte de las mujeres, mientras que el aprendizaje de la masculinidad llevaría implícito un mayor desarrollo de la autonomía y de la conciencia de los propios intereses.

3- Existen cuestiones en conflicto dentro de las relaciones afectivas vinculadas con el sistema tradicional de género, como la asimetría en el intercambio emocional o el déficit de autoridad y poder de las mujeres. Esto significa que el mantenimiento de los roles de género clásicos puede tener un efecto negativo en la dinámica de una relación íntima entre mujer y hombre, teniendo en cuenta que la igualdad y la reciprocidad pueden formar parte de las expectativas personales en un contexto democrático en el que se han vivido transformaciones profundas en cuanto al papel de las mujeres en la sociedad y a su condición jurídica.

\subsection{El estudio cualitativo}

La investigación se realiza en el marco metodológico de la Antropología Social y, tiene en consecuencia un carácter esencialmente cualitativo, otorgando una importancia fundamental a la entrevista en profundidad y abierta.

El acceso al discurso directo de personas sobre su propia experiencia afectiva a través de entrevistas en profundidad cumple el objetivo de obtener descripciones amplias de la propia experiencia de los/las informantes, así como del valor y significados que les asignan, poniendo de relieve que la realidad social y específicamente humana se construye, se aprende, se organiza e interpreta; en ella confluyen no sólo intereses y funciones, sino fundamentalmente creencias, sistemas de valores y significados culturales (Hammersley y Atkinson, 1988). De esta forma, se ha considerado necesario el acercamiento al tema del amor a través de la conversación, pues es en el lenguaje en interacción donde se expresa estructuralmente el contexto, haciendo visible el sistema de significados y valores que vertebra el discurso (Whorf, 1999).

El análisis del discurso se ha planteado en este trabajo, no como ejercicio meramente lingüístico ni informacional, sino centrado en el nivel social/hermenéutico del discurso, siguiendo la visión de Alonso (1998: 189-220). En este sentido, resulta relevante para este análisis hacer hincapié en el proceso de construcción de las relaciones sociales a partir de un determinado contexto, dando preferencia a esta visión dinámica de la realidad en la tarea de aproximación empírica a las relaciones de pareja, y admitiendo la utilidad de la entrevista abierta para obtener información sobre "cómo los sujetos diversos actúan y reconstruyen el sistema de representaciones sociales en sus prácticas individuales” (Alonso, 1998: 72). Así, tras la selección de información a partir de las categorías sobre las que se construía el análisis, se pasó a su organización e interpretación poniendo en relación aspectos de la realidad 
macroestructural con aquéllos que se manifiestan en las realidades concretas de las personas entrevistadas, con el objetivo de descubrir estructuras estables de sentido.

Además, se ha adoptado un enfoque interdisciplinar en coherencia con el objeto de estudio (en cuyo conocimiento tienen una importancia fundamental las aportaciones de la Psicología) y con las peculiaridades de los Estudios de Género.

\subsection{Entrevistas y participantes}

La información cualitativa a la que se hace referencia en este artículo proviene de 46 entrevistas en profundidad de carácter biográfico realizadas a 23 mujeres y 23 hombres, de entre 18 y 77 años, manteniendo una representación relativamente proporcional de las franjas de edad establecidas (de 18 a 30 años, de 31 a 49 años, de 50 años en adelante). Todas ellas eran españolas con excepción de una mujer, de origen latinoamericano, y de un hombre, de origen italiano, aunque ambos residen indefinidamente en España desde hace más de cinco años.

Para resolver la cuestión del acceso a este grupo de informantes, se diseñó un cuestionario con preguntas en su mayoría de respuesta cerrada que incluía una casilla donde se solicitaba un teléfono o e-mail de contacto a la persona que accediera a colaborar con el proyecto mediante una entrevista. Este cuestionario se distribuyó en 12 clases universitarias de diferentes centros (Universidad Miguel Hernández de Elche, Universidad de Alicante y UNED). Se trata, por tanto, de una población escogida a partir de su vinculación con la universidad y no por criterios geográficos o económicos. No obstante, todas estas personas residen en la provincia de Alicante y muestran unas características socioeconómicas similares que hacen que puedan ser adscritas a lo entendido como clase media.

Por otro lado, fue imprescindible contar con personas cuya situación fuera representativa de la diversidad presente hoy en la sociedad en cuanto a las diferentes formas que asumen las relaciones afectivas. Es por ello que entre los/las participantes se hallan personas casadas, separadas, con parejas de hecho, con hijos y sin hijos, personas con pareja sin convivencia, e incluso, sin relación de pareja en la actualidad. También constituía un requisito de la investigación el haber tenido experiencias afectivas de pareja (no necesariamente en el momento de la entrevista) de, al menos, un año.

\section{El conflicto en torno a la desigualdad emocional}

Al analizar los conflictos afectados transversalmente por el sistema de género, comprobamos la vigencia de la afirmación de Hite (1988: 80) que contempla la desigualdad emocional como principal elemento perturbador del amor en las relaciones heterosexuales, al 
comprobar que sólo el $19 \%$ de las mujeres participantes en su estudio describían relaciones emocionales de igualdad.

En esta investigación, se aprecian asimismo dinámicas similares que confirman la tendencia descrita por Hite, notando que uno de los conflictos aparecidos con mayor frecuencia en las entrevistas tiene que ver con la interacción comunicativa y emocional entre la mujer y el hombre en su relación de pareja. El testimonio que se recoge a continuación pertenece a una mujer que acaba de romper una relación de cinco años que describe como tormentosa, en la que ella se queja principalmente de la falta de implicación de su expareja en aspectos comunes, describiendo situaciones en las que su necesidad de comunicación en determinados momentos se ve frustrada por una postura menos favorable al acercamiento por parte de él.

"Cuando discutíamos él pasaba de mí, y claro, yo pues me ponía fatal, porque veía que me ignoraba, pensaba que no me quería, él decía que me ponía de una forma que no sabía cómo resolver, podíamos estar luego un día sin hablarnos, al final tenía que ir yo a hablar,... hasta que me cansé" (Mujer, 33 años, soltera).

La descripción de situaciones en las que las mujeres desean una mayor expresión de cariño y apoyo emocional por parte de sus parejas se produce de hecho de forma generalizada. En este sentido, los resultados aquí descritos coinciden con los presentados recientemente en otros estudios internacionales (Duncombe y Marsden, 1993; Fishman, 1982; Benjamin, 2011) que concluyen que la mayor responsabilidad femenina sobre la gestión de las emociones en la esfera privada se da conjuntamente con una extendida insatisfacción de las mujeres con respecto a la implicación emocional de sus parejas masculinas. Por ejemplo, en el siguiente testimonio una mujer casada manifiesta su deseo de un mayor interés por parte de su marido por las actividades que ella realiza como parte de sus aficiones en su tiempo libre. En este caso, la mujer hace alusión a una relación estable que dura alrededor de veinte años (siendo la única considerada por ella como verdaderamente importante en su vida y en la que este conflicto no iría unido a un pensamiento de ruptura.

"Que yo le digo a mi marido esto y no me hace ni caso porque no le interesa porque es verdad que
no le interesa, y a veces echas de menos eso, por ejemplo, he ido a alguna carrera y he subido al
podium y no ha estado porque él pasa" (Mujer, 40, casada).

También los hombres que describen situaciones de conflicto que dieron lugar a la ruptura de su relación de pareja otorgan una especial importancia a la cuestión emocional. Algunos testimonios describen situaciones en las que la no expresión de sentimientos por su parte genera un conflicto que se acentúa por la misma dificultad que plantea esta actitud a la hora de resolverlo. En el primer caso que se expone, el informante de mediana edad habla de una relación que duró nueve años, confesando no haber sido consciente de la gravedad del problema que suponía para su expareja su falta de expresión de afecto, a la que ella aludió al romper la relación. En el segundo ejemplo, el entrevistado habla concretamente del conflicto vivido en su última relación de pareja provocado por su distanciamiento emocional, aunque a 
lo largo de la conversación se señala que este aspecto también apareció ligado a problemas en sus relaciones anteriores.

"Por no saber transmitir que no era indiferente..., [...] muchas veces el conflicto no se solucionaba sino que se agravaba, claro, iba yo como a pedir perdón, porque yo veía a mi novia destrozada, hecha polvo, y encima iba yo como lamentándome de que yo era una víctima, y lo que hacía era rematar la faena” (Hombre, 40 años, soltero).

"Conflictos tuvimos fundamentalmente porque ella exigía, a mi modo de ver, de mí unas manifestaciones afectivas, unas respuestas afectivas, que yo no tenía, me pedía más en ese sentido de lo que yo podía dar, entonces eso me agobiaba y hacía que yo me retrajese más todavía y ella se agobiaba a su vez cada vez más y eso generaba un círculo vicioso" (Hombre, 42 años, soltero).

En otros casos, son las diferencias en las expectativas de hombres y mujeres en cuanto a la forma de compartir la vida lo que plantea desencuentros derivados del choque entre un modo marcadamente individualista, que las mujeres asocian con lo masculino, y otro definido desde la dependencia emocional, que tiende a interpretarse como más propio de las mujeres. En los siguientes discursos, esta cuestión se plantea como foco de conflictos, aunque si bien en el primer caso forma parte de los aspectos negativos que dieron lugar a la ruptura de la pareja, en el segundo aparece simplemente como una queja, asociándose a su vez con los estereotipos de género que se asumen sin cuestionamientos.

"Era muy individualista, y yo ahí era más inmadura y reclamaba demasiado que estuviera conmigo y él no quería. [...] yo no sé por qué, tenía yo una falta de que me quisieran, no sé por qué motivo, como querer siempre estar con él, siempre con él” (Mujer, 34 años, soltera).

"La mujer siempre tiende a ser más conservadora y en ese aspecto más tradicional, y un poquito más acaparadora, no sé, ve la relación siempre juntos, hay que hacerlo todo juntos, ahora ya somos dos y se cuenta para todo con los dos y yo creo que eso, no sé, [...] la mayoría de los hombres siempre se quejan de eso, yo hablo con muchos amigos casados y me dicen todos lo mismo" (Hombre, 35 años, casado).

Se ha de notar también que, al analizar las diferencias entre mujeres y hombres en el aspecto emocional, se aprecia todavía una tendencia a considerar por parte de ambos la atención y el cuidado como algo que aparece naturalmente en la actitud femenina hacia el hombre, siguiendo el esquema de género en el que la mujer se conceptualiza como "dadora" en lo referente al amor. La fuerza con la que algunas mujeres asumen las responsabilidades emocionales se puede manifestar incluso paralelamente a una valoración negativa de esta misma actitud cuando es protagonizada por los hombres, reflejando la interiorización del mismo esquema de género en el que lo prescrito para hombres y mujeres tiende a mostrar cualidades antagónicas, y del que se genera una contradicción implícita entre masculinidad y cuidado.

La unión simbólica de lo femenino al mundo emocional se manifiesta además en la importancia que puede tener para el hombre el vínculo con la mujer en el hecho de descubrirse a sí mismo. En el acercamiento a lo femenino, de este modo, el hombre puede mostrarse 
desinhibido en relación con sus sentimientos y expresar con libertad sus necesidades, algo que el modelo tradicional de masculinidad negaría o concebiría como "inapropiado" para el sexo masculino en ámbitos fuera de la relación de la pareja.

\begin{abstract}
"Nunca me ha gustado estar solo, siempre la presencia de una mujer a mi lado es como un complemento que yo tenía a mi forma de ser, yo soy muy romántico, soy muy sentimental, me emociono y necesito esa pareja que esté a mi lado para poder sacarlo, yo no lo saco con mis amigos, saco mi forma de ser pero superficialmente, por dentro de mí yo lo saco con una pareja a mi lado" (Hombre, 50 años, casado).
\end{abstract}

Merece comentario igualmente la presencia de diferencias estructurales por edad en la forma en que se intercambian los cuidados en la pareja. Al formar parte el cuidado de las tareas tradicionalmente asignadas a las mujeres, en el grupo de mayor edad se puede encontrar una clara desigualdad en este aspecto sin que su presencia vaya necesariamente unida al conflicto. La asimetría en estos casos se apoya en la asignación diferencial de deberes de hombres y mujeres hacia la familia dentro del sistema tradicional. Es el grupo de mediana edad el que mostraría una mayor presencia de conflictos ligados a la desigualdad.

No obstante, aunque los discursos de las personas más jóvenes reflejan la asimilación de valores como la igualdad y la libertad en el ideal de relación amorosa, también es frecuente encontrar en ellos la influencia de los valores tradicionales que asocian la identidad femenina con la actitud altruista hacia los demás y la predisposición al cuidado. Se recogen aquí dos testimonios de mujeres jóvenes, con relaciones de pareja descritas como satisfactorias e igualitarias, en los que puede observarse una experiencia del amor orientada a la satisfacción de las necesidades del otro, o determinada por el hecho de que la pareja constituya un elemento fundamental de la vida.

\footnotetext{
"Para mí el amor es considerar a la persona que está contigo lo más importante del mundo, para mí es lo más importante del mundo, a mí me preocupa que esa persona esté bien, que se encuentre bien en todos los ámbitos, de salud, mental, en todo, que esté a gusto con lo que hace el día a día, que sea feliz, a mí me importa que esa persona sea feliz, conmigo y con todo lo que le rodea, con su familia, con su trabajo, con todo" (Mujer, 24 años, con pareja).

"Sí que es verdad que cuando llevaba un tiempo sola, veía a los demás y decía, yo también quiero, me siento mucho mejor en pareja [...] yo por lo menos sentiría que me falta algo" (Mujer, 18 años, con pareja).
}

Así, se expresa en muchas ocasiones una contradicción entre la forma en que las mujeres experimentan una vida social/profesional con total igualdad con respecto a sus familiares y compañeros varones y la forma en que asumirán su vida íntima en el contexto de la pareja, notando que la interacción informal de hombres y mujeres puede estar más influida por las ideologías heredadas e incorporadas en la propia personalidad que por los valores que dan sentido a un sistema democrático moderno. 


\section{La desigualdad emocional en la resolución del conflicto}

El contemplar la expresión emocional en el ámbito de la pareja heterosexual como una cuestión atravesada por el género nos lleva también a valorar la presencia de diferentes estrategias de resolución de conflictos como fenómeno unido a la desigualdad, entendiendo que la unión simbólica de las estrategias relacionales a las identidades de género puede llegar a producir comportamientos femeninos más sumisos y/o empáticos y comportamientos masculinos más asertivos y/u orientados a la defensa de los propios intereses. En relación con este aspecto, este trabajo sí detecta diferencias de género importantes en el modo de afrontar los problemas más graves sobre los que se ha tenido conocimiento a través de las entrevistas. En general, aunque las diferencias en el modo de afrontar un conflicto se interpretan por parte de hombres y mujeres como algo vinculado con la propia personalidad, la tendencia a delimitar el espacio individual y a actuar en defensa de las propias necesidades aparece con más fuerza en los discursos masculinos.

"Sí creo que son cosas importantes, mi ocio, mi tiempo libre, y eso no lo pongo a discusión. Yo pienso que es algo que me pertenece, que es mío" (Hombre, 35 años, casado).

En el caso de las mujeres, aparece de forma más contundente la necesidad de comunicación verbal en el contexto del conflicto, muchas veces, no exclusivamente orientada a exponer las propias necesidades o intereses, sino a la búsqueda de entendimiento inmediato de la pareja y al establecimiento de un clima apacible.

"Cuando tenemos un conflicto a mí me gusta resolverlo rápidamente, es decir, no suelo guardar silencio, ni me enfado y no te hablo hasta dentro de dos días, ni cosas de ese tipo, no me gustan nada de nada, entonces si tenemos un conflicto me gusta hablarlo, o sea, ya, decir bueno, qué pasa, pues bueno, pues ya está, lo siento o lo que sea, aunque tuviera un poco de enfado, pero hablarlo ya y él en eso es un poco más rencoroso, si hay algo que realmente le ha molestado, le ha molestado, no habla, o te habla poco, y se queda con la cosilla ahí hasta que dentro de 2 o 3 días te la suelta" (Mujer, 28 años, con pareja).

Las diferencias en las posturas de hombres y mujeres con respecto a la comunicación producen en consecuencia acercamientos marcados por un patrón de desigualdad emocional, viendo que la inversión de energía y esfuerzo en la relación para lograr la reconciliación y el bienestar es muy a menudo mayor en la mujer. En el siguiente testimonio una mujer describe cómo vivía las discusiones y los acercamientos en una relación de pareja ya terminada que la llevó a recibir tratamiento psicológico. Su relato pone de manifiesto el esfuerzo de la mujer por restaurar un clima de bienestar y conservar la relación a través de la modificación de su comportamiento, adaptándose a los deseos de su expareja.

“Él solía irse, entonces yo me hundía totalmente y cuando él quería volvía, yo me tenía que recomponer y decir, venga, si estoy en esta actitud él no va a querer volver, o sea, tengo que 
intentar estar bien, porque si lloro, él va a decir, «ves, como estás llorando me voy». Entonces me animaba yo sola, intentaba estar mejor, y entonces él, si me veía que yo había cambiado, a lo mejor, o yo le llamaba, o él... a lo mejor, volvía" (Mujer, 34 años, soltera).

De manera inversa, la distancia emocional, en contraste con la disposición a la conversación en el momento del conflicto, aparece con mayor frecuencia en el comportamiento masculino, dentro de la heterogeneidad que caracterizaría las respuestas de los hombres y sus posturas con respecto a las cuestiones tratadas en la investigación. Esta actitud puede estar presente especialmente en los conflictos que plantean para la pareja una mayor gravedad y que, con frecuencia, acaban en la ruptura de la relación, como en los ejemplos que siguen.

"El que me dejara llorando tantas veces, esos fueron los momentos peores para mí, el que los momentos que discutíamos y no nos entendíamos, el que fuera tan frío en ese sentido y no se acercara a mí a un mínimo encuentro" (Mujer, 33 años, soltera).

"Estaba frío porque no quería mostrar sentimientos, después en casa me derrumbé" (Hombre, 38 años, soltero).

El conflicto marca así un momento clave en la relación de pareja heterosexual que a menudo refuerza la autoridad masculina y exige la adaptación de la mujer para conservar el vínculo. La adaptación aparece de hecho con relativa frecuencia en los discursos de las mujeres como modo de rebajar el conflicto. Este comportamiento puede relacionarse con la capacidad de autoridad y autoafirmación de cada una de las partes en la dinámica de la relación de pareja. El hecho de que las mujeres acaben adaptándose a la relación asumiendo el comportamiento que se espera de ellas puede ser visto como resultado de un déficit de autoridad femenina dentro de la relación de pareja. A continuación, se han recogido dos testimonios de mujeres con relaciones estables, la primera de ellas viuda y emparejada con un hombre también viudo desde hace ocho años, y la segunda casada diez años con el hombre que ha sido su pareja desde los veinte. En ambos casos, los discursos reflejan una menor autoridad femenina con respecto a los hombres, cuyas decisiones prevalecerían en la dinámica de la relación, siendo aceptadas por ellas como modo de evitar el conflicto que resultaría del choque de intereses.

"Yo muchas veces le digo «podemos casarnos» y él contesta enseguida, «pero si vivimos bien así, para qué queremos casarnos». O sea que ya, yo no quiero casarme" (Mujer, 74 años, viuda y con pareja).

"A mí me gusta hacer gimnasia, yo salgo a correr, salgo a andar con el perro, y él no, él es muy pasivo, le gusta el sofá, fuma mucho, yo fumo, un poco. Entonces siempre tengo que tirar, que tirar, que tirar, pero bueno, cuando él libra, porque él trabaja un fin de semana sí y otro no, entonces tenemos poco tiempo, el fin de semana que él libra yo hago lo que él diga, lo que le guste. Quiere ir al cine, al cine, a veces dice, vamos a pasear, bueno, hasta donde él llegue" (Mujer, 40, casada). 
Además, en muchas ocasiones se puede observar que, ante un conflicto o aspecto causante de insatisfacción, muchas mujeres tienden a aceptar determinados tópicos y estereotipos de género que normalizarían la desigualdad, justificando así la menor implicación de sus parejas, fenómeno que reforzaría los roles de género en la dinámica de la relación. Esto significa que la desigualdad de género puede manifestarse simultáneamente como origen del conflicto y como estrategia para solucionarlo.

"A mí me ayudó el libro ese [Los hombres son de Marte y las mujeres de Venus] mucho a
entender ciertas cosas, porque explica muchas situaciones que a mí me pasan, que quieres hablar
y ver que él no quiere, «y es que no me quieres, no me quieres porque si no, arreglarías el tema»,
«ahora no quiero hablar porque nos vamos a enredar más aún», y luego lo entiendes, y bueno,
será él así [...]. Es que hablamos lenguajes diferentes, es que somos diferentes en todo" (Mujer,
40, casada).

Por otro lado, en las parejas cuya dinámica y/o ideología están más marcadas por la igualdad, la presencia de conflictos, así como el modo en que se resuelven, no presenta patrones de género significativos. En estos casos, el acercamiento suele estar propiciado por una valoración consciente por parte de ambos de la situación y de los comportamientos que pueden haber causado el malestar.

\section{Conclusiones}

En resumen, este análisis ha detectado la presencia de un sufrimiento mayor ligado a rupturas y conflictos en las/los participantes de mediana edad, en dinámicas de pareja que reflejan una cierta incompatibilidad entre el sistema tradicional de valores de género y las nuevas condiciones de vida que permiten que las relaciones afectivas puedan construirse sobre el ideal de igualdad y reciprocidad. De ahí que se pueda concluir que la excesiva rigidez de los estereotipos de género tradicionales no sólo no reduce el conflicto en las relaciones entre los hombres y las mujeres actualmente, sino que lo acrecienta.

A partir de los resultados de este estudio, resulta especialmente significativo el conflicto derivado de la interacción emocional de la pareja. La desigualdad emocional constituye uno de los principales conflictos que afectan a las relaciones de pareja en las que hombre y mujer adquieren iguales responsabilidades laborales y económicas. En estas condiciones, las expectativas de reciprocidad de las mujeres chocarían a menudo con una experiencia cotidiana que les haría asumir una mayor responsabilidad alrededor del trabajo emocional que conlleva el cuidado del otro, así como del mantenimiento de un clima emocional adecuado en el desarrollo de la convivencia, fenómeno a su vez relacionado con la pervivencia de un sistema de valores en el que las actitudes orientadas al cuidado y al amor son asignadas al comportamiento femenino. También se ha destacado que, aun cuando esta desigualdad es naturalizada a partir de creencias en las diferencias esenciales entre hombres y mujeres, la falta 
de entendimiento y apoyo recíproco produce sentimientos profundos de frustración en muchas mujeres, lo que acaba socavando el bienestar conyugal.

Con respecto a la resolución del conflicto se ha podido observar que, en las relaciones no igualitarias en el plano emocional y ante la existencia de un conflicto grave, es principalmente la intervención de la mujer, o su adaptación a las condiciones que se le plantean, la estrategia que va a permitir mantener la relación de pareja, así como reestablecer un clima emocional que proporcione bienestar. Esta actitud revela que en muchos casos el conflicto puede resolverse (o evitarse) mediante el reforzamiento de los roles de género tradicionales. Asimismo, pone de manifiesto la existencia de dos respuestas mayoritarias ante el conflicto derivado de la desigualdad: el fortalecimiento de los roles de género o la separación.

La alta probabilidad de que el conflicto personal entre hombre y mujer se resuelva mediante el reforzamiento de los roles clásicos, reafirmando, por ejemplo, el distanciamiento emocional y la autoridad en él, y la adaptación y aceptación de una mayor responsabilidad sobre la gestión emocional en ella, se da porque estas actitudes estarían culturalmente legitimadas, serían aprendidas socialmente y formarían parte del único código de conducta conocido para regular los conflictos propios de las relaciones de pareja. Esta tendencia pone de manifiesto la falta de recursos culturales orientados a la resolución pacífica de conflictos, haciendo visible la centralidad que todavía tiene el respeto a un orden de género en la organización y funcionamiento de las relaciones de pareja.

\section{Bibliografía}

- Alonso, Luis Enrique (1998): La mirada cualitativa en sociología. Una aproximación interpretativa. Madrid: Editorial Fundamentos.

- Bauman, Zygmunt (2005): Amor líquido. Acerca de la fragilidad de los vínculos humanos. Madrid: Fondo de Cultura Económica.

- Beck, Ulrich y Beck-Gernsheim, Elizabeth (2006): El normal caos del amor. Barcelona: Paidós Contextos - El Roure.

- Benjamin, Orly (2011): “Gender and contesting models of love: Ambivalence as Silencing in Israeli intimate relationships". Paper presented at the I Global Conference: Gender and Love, Mansfield College, Oxford (United Kingdom), September, [en línea], Disponible en: http://www.inter-disciplinary.net/wp-content/uploads/2011/08/benjamingpaper.pdf [14/12/2012].

- Bericat Alastuey, Eduardo (2000): “La sociología de la emoción y la emoción en la sociología”. En: Papers, nº 62, pp. 145-176.

- Bourdieu, Pierre (2000): La dominación masculina. Barcelona: Anagrama. 
- Curtis, Richard F. (1986): "Household and Family in Theory on Inequality". En: American Sociological Review, vol. 51, nº 2, Apr., pp. 168-183.

- Duncombe, Jean y Maersden, Dennis (1993): "Love and Intimacy: The Gender Division of Emotion and «Emotion Work». A Neglected Aspect of Sociological Discussion of Heterosexual Relationships”. En: Sociology, May, vol. 27, pp. 221-241.

- Fishman, Pamela M. (1982): "Interaction: The Work Women Do". En: Social Problems, no 25, pp. 397-406.

- Giddens, Anthony (1995): La transformación de la intimidad: sexualidad, amor y erotismo en las sociedades modernas. Madrid: Cátedra.

- Hammersley, Martyn y Atkinson, Paul (1994): Etnografía. Métodos de investigación. Barcelona: Paidós.

- Hite, Shere (1988): Mujeres y amor. Nuevo informe Hite. Barcelona: Plaza \& Janes Editores.

- _ _ (1995): Informe Hite sobre mujeres y amor. Barcelona: Paidós.

- Hochschild, Arlie R. (1979): "Emotion Work, Feeling Rules, and Social Structure". En: American Journal of Sociology, vol. 85, nº 3, Nov., pp. 551-575.

- ___ (1997): The Time Bind. New York: Metropolitan Books.

- Izquierdo, María Jesús (1998): El malestar en la desigualdad. Madrid: Cátedra.

- Jamieson, Lynn (1999): "Intimacy Transformed? A Critical Look at the «Pure Relationship». En: Sociology, August 1999, vol. 33, n. 3, pp. 447-494.

- Jónasdóttir, Anna G. (1993): El poder del amor: ¿le importa el sexo a la democracia? Madrid: Ediciones Cátedra.

- Rebollo Catalán, Mán Ángeles (2006): "Emociones, género e identidad: la educación sentimental”. En: Ma Ángeles Rebollo (coord.): Género e interculturalidad: educar para la igualdad. Madrid: La Muralla, pp. 217-244.

- Saltzman, Janet (1992): Equidad y género. Una teoría integrada de estabilidad y cambio. Madrid: Ediciones Cátedra.

- Sanz, Josefina (2000): Los vínculos amorosos. Amar desde la identidad en la Terapia de Reencuentro. Barcelona: Kairós.

- Verdú Delgado, Ana Dolores (2012): "El amor y las relaciones de pareja desde la perspectiva de género. Un estudio interdisciplinar”. Tesis Doctoral. Universidad Miguel Hernández de Elche.

- Whorf, Benjamin Lee (1999): "Un modelo indio-americano del universo". En: Eduardo de Bustos: Filosofía del lenguaje. Madrid: UNED, p. 185. 